\title{
ANÁLISE DOS REGISTROS DE USUÁRIOS DO PROGRAMA HIPERDIA
}

Marcia dos Santos Casaril Cargnin1, Jonathan da Rosa², Isabel Cristina Echer ${ }^{3}$

`Enfermeira. Mestre em Enfermagem. Universidade Regional Integrada do Alto Uruguai e das Missões. Rio Grande-RS-Brasil. Enfermeiro. Instituto Municipal de Estratégia de Saúde da Família. Porto Alegre-RS-Brasil.

${ }^{3}$ Enfermeira. Doutora em Clínica Médica. Universidade Federal do Rio Grande do Sul. Porto Alegre-RS-Brasil.

RESUMO: Estudo de cunho documental, epidemiológico descritivo, teve por objetivo avaliar a efetividade dos registros das fichas de cadastro de usuários do Sistema de Cadastramento e Acompanhamento de Hipertensos e Diabéticos - HIPERDIA de um município do Rio Grande do Sul-Brasil. Os dados relativos a 637 cadastros de 17 micro áreas foram compilados, no período de agosto de 2010 a dezembro de 2011 e analisados por meio do programa BIOESTAT ${ }^{\circledR}$ versão 4.0. Destaca-se entre os resultados maior prevalência de hipertensos; tabagismo, sedentarismo e sobrepeso/obesidade como fatores de risco; infarto agudo do miocárdio, acidente vascular cerebral e doença renal como complicações das doenças. O expressivo não preenchimento de campos obrigatórios e de interesse epidemiológico pode prejudicar melhor caracterização dos usuários e o planejamento de ações de prevenção e reorganização dos serviços de saúde.

DESCRITORES: Cadastro; Diabetes mellitus; Hipertensão; Efetividade.

\section{ANALYSIS OF THE RECORDS OF USERS OF THE 'HIPERDIA' PROGRAM}

\begin{abstract}
This documental, descriptive and epidemiological study aimed to evaluate the quality of the record taking in the registration forms of users of the Hypertensives and Diabetics Registration and Monitoring System (known in Portuguese as HIPERDIA) in a municipality in the state of Rio Grande do Sul (RS), Brazil. The data relating to 637 records from 17 micro-areas were compiled, for the period August 2010 - December 2011 and were analyzed using the $\mathrm{BIOESTAT}^{\circledR}$ program, version 4.0. Among the results, the following stand out: higher prevalence of hypertensives; smoking, sedentarism and overweight/obesity as risk factors; and acute myocardial infarction, cerebrovascular accidents and kidney disease as complications of the diseases. The significant failure to fill out areas which are obligatory and of epidemiological interest may hinder better characterization of the service users and the planning of preventive actions and reorganization of the health services.
\end{abstract}

DESCRIPTORS: Records; Diabetes mellitus; Hypertension; Effectiveness.

\section{ANÁLISIS DE LOS REGISTROS DE USUARIOS DEL PROGRAMA HIPERDIA}

RESUMEN: Estudio documental, epidemiológico descriptivo, que tuvo por objetivo evaluar la efectividad de los registros de fichas de usuarios del Sistema de Registro y Acompañamiento de Hipertensos y Diabéticos - HIPERDIA de un municipio del Rio Grande do Sul, Brasil. Los datos referentes a 637 registros de 17 micro áreas fueron compilados en el periodo de agosto de 2010 a diciembre de 2011 y analizados por medio del

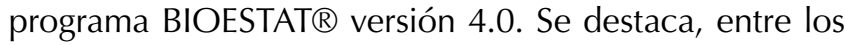
resultados, mayor prevalencia de hipertensos; tabaquismo, sedentarismo y sobrepeso/obesidad como factores de riesgo; infarto agudo del miocárdio, accidente vascular cerebral y enfermedad renal como complicaciones de las enfermedades. El expresivo no relleno de campos obligatorios y de interés epidemiológico puede perjudicar una buena caracterización de los usuarios y el planeamiento de acciones de prevención y reorganización de los servicios de salud.

DESCRIPTORES: Registro; Diabetes Mellitus; Hipertensión; Efevtividad.
Autor Correspondente:

Marcia Casaril dos Santos Cargnin

Universidade Regional Integrada do Alto Uruguai e das Missões

Rua Pedro Alvares Cabral, 36 - 98410-000 --Taquaruçu do Sul-RS-Brasil

E-mail: marciacasaril@hotmail.com
Recebido: 08/01/2013 Finalizado: 14/11/2013 


\section{INTRODUÇÃO}

As Doenças Crônicas Não Transmissíveis (DCNT), entre elas Hipertensão Arterial Sistêmica (HAS) e Diabetes Mellitus (DM), ganharam destaque nos últimos anos pelo crescente número de indivíduos que as apresentam, agravado pelo aumento dos já diagnosticados, que não conseguem fazer o controle adequado da doença. Estes agravos à saúde são responsáveis por $66 \%$ dos anos de vida perdidos e por complicações clínicas relevantes, que implicam em mortes prematuras, incapacidades para o trabalho e gastos públicos em crescente expansão ${ }^{(1)}$, incluindo importante repercussão na vida da população.

A HAS e o DM, além de serem evitáveis, compartilham muitos fatores de risco comuns. No ano de 2010, no Brasil, o DM foi responsável por $4,7 \%$ das mortes, enquanto a HAS $4,0 \%$, só perdendo para as enfermidades cerebrovasculares, isquêmicas do coração, influenza, pneumonia e pelas agressões e homicídios ${ }^{(2)}$.

Neste sentido, devido à necessidade de um instrumento que permita cadastrar e acompanhar os usuários de serviços de saúde e gerar informações do desempenho e dos resultados clínicos, o Ministério da Saúde criou em 2002 o Sistema de Cadastramento e Acompanhamento de Hipertensos e Diabéticos (HIPERDIA). Este apresenta como propósito detectar e vincular os portadores desses agravos às unidades de saúde e identificar, precocemente, complicações relacionadas às doenças, efetuando tratamento adequado, favorecendo atividades de prevenção e de reorganização dos serviços de saúde ${ }^{(3)}$.

Entretanto, este permite apenas visualizar dados gerais da situação de saúde dos usuários e muitas Unidades Básicas de Saúde (UBS) não conseguem abordar a população com efetividade $^{(4)}$. Deste modo, é preciso investigar a dinâmica epidemiológica das doenças para conhecer a distribuição, a exposição e os fatores que influenciam os riscos, subsidiando o processo de prevenção e acompanhamento dessas doenças nas comunidades.

Este artigo originou-se a partir do desenvolvimento de ações de extensão universitária junto a um município no norte do Estado do Rio Grande do Sul (RS) - Brasil, no momento em que surgiu a necessidade de conhecer a realidade local de hipertensos e diabéticos cadastrados no programa HIPERDIA. Para isso, propôs-se o desenvolvimento da pesquisa com o objetivo de avaliar a efetividade dos registros das fichas de cadastro de usuários de um programa HIPERDIA.

\section{MÉTODO}

Tratou-se de estudo documental, de caráter quantitativo, do tipo epidemiológico descritivo, realizado em um município da região noroeste do Estado do Rio Grande do Sul, Brasil, com área territorial de $364 \mathrm{Km} 2$ e população estimada de 7.878 habitantes $^{(5)}$. O município conta com uma UBS e uma Estratégia de Saúde da Família (ESF), ambas localizadas na zona urbana. Seu território estava dividido em 20 micro áreas de saúde.

A população do estudo foi constituída de pessoas cadastradas no programa HIPERDIA da UBS e da ESF do município, no período de agosto de 2010 a dezembro de 2011. A coleta de dados ocorreu por meio da compilação das fichas de cadastro do programa HIPERDIA arquivadas na Secretaria Municipal de Saúde, em um formulário elaborado pelos pesquisadores. Foram analisados 637 cadastros do programa, correspondentes a 17 das 20 micro áreas existentes no município. Três micro áreas não foram incluídas no estudo por não apresentarem, no período da coleta, Agentes Comunitários de Saúde para atualizar os cadastros.

O critério de inclusão foi de o usuário possuir cadastro atualizado no programa HIPERDIA. As variáveis selecionadas para a análise foram categorizadas em oito grupos, segundo dados da Ficha do Programa HIPERDIA, conformeQuadro 1.

Para organização e análise dos dados utilizou-se Planilha do Programa Windows

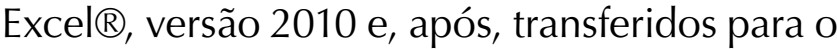
programa BIOESTAT® versão 4.0, analisando-se frequência e percentual.

Os princípios éticos e legais da resolução $466 / 2012$ foram respeitados e o projeto aprovado pelo Comitê de Ética e Pesquisa da Universidade Regional Integrada do Alto Uruguai e das Missões, sobre registro CAAE n. 0020.0.284.000-10. 


\begin{tabular}{|l|l|}
\hline Grupo de variáveis & Dados da ficha do programa HIPERDIA \\
\hline Cabeçalho da ficha & $\begin{array}{l}\text { Nome da unidade saúde; Código SAI/SUS; Número do prontuário do } \\
\text { paciente. }\end{array}$ \\
\hline Identificação do usuário & $\begin{array}{l}\text { Nome usuário; da mãe; do pai; Data nascimento; Sexo; Raça/Cor; Esco- } \\
\text { laridade; Nacionalidade; País origem; Data naturalização; Número por- } \\
\text { taria; Unidade Federativa e nome do município de nascimento; Situação } \\
\text { familiar/conjugal; Número cartão Sistema Único de Saúde. }\end{array}$ \\
\hline Documentos gerais & $\begin{array}{l}\text { Título eleitor; Zona título eleitor; Série título eleitor; Número, Série, Data } \\
\text { da emissão e Unidade Federativa da Carteira de Trabalho e Previdência } \\
\text { Social; Cadastro de Pessoa Física; Programa de Integração Social/Pro- } \\
\text { grama de Formação do Patrimônio do Servidor Público.. }\end{array}$ \\
\hline Documentos obrigatórios & $\begin{array}{l}\text { Identidade; Complemento da identidade; Órgão emissor identidade; } \\
\text { Unidade Federativa da identidade; Data Emissão identidade; Certidão; } \\
\text { Tipo certidão; Nome cartório; Livro; Folha; Termo; Data emissão. }\end{array}$ \\
\hline Endereço & $\begin{array}{l}\text { Tipo do logradouro; Nome do logradouro; Número; Complemento; Bair- } \\
\text { ro; Código de endereçamento postal; Telefone. }\end{array}$ \\
\hline Dados Clínicos do paciente & $\begin{array}{l}\text { Pressão Arterial Sistólica; Pressão Arterial Diastólica; Fatores risco ou } \\
\text { doenças concomitantes; Antecedentes familiares/cardiovasculares; Dia- } \\
\text { betes Tipo I; Diabetes Tipo II; Tabagismo; Sedentarismo; Sobrepeso/Obe- } \\
\text { sidade; Hipertensão Arterial Sistêmica e Estratificação do risco e quali- } \\
\text { ficação de prognóstico de Pressão Arterial; Índice de Massa Corpórea; } \\
\text { Glicemia; Presença de Complicações (Infarto agudo do miocárdio, Out- } \\
\text { ras coronariopatias, Acidente vascular cefálico, Pé diabético, Amputação } \\
\text { por diabetes, Doença renal); Tratamento: Medicamentoso; Outros me- } \\
\text { dicamentos; Uso de Insulina. }\end{array}$ \\
\hline Registro atendimento & Data da Consulta; Assinatura do responsável. \\
\hline
\end{tabular}

\section{RESULTADOS}

Dos 637 cadastros do programa todos os dados de identificação dos usuários apresentaram o nome completo do usuário. Verificou-se que na área destinada ao cabeçalho da ficha, que compreende informações como nome da unidade de saúde, código SIA/SUS e número do prontuário, 96(15\%) dessas, referentes à unidade, foram preenchidos. Um número reduzido de fichas apresentou o número do cadastro do cartão do Sistema Único de Saúde (SUS).

Os dados de data de nascimento, nome da mãe e nome do pai houve média de 95\% de dados informados. No campo nacionalidade $621(97,4 \%)$ das fichas foram preenchidas como brasileiros; por outro lado, campos referentes ao país de origem e data de naturalização, $33(52,4 \%)$ e $58(9 \%)$ apresentavam o dado. Em 63(100\%) não pode ser identificado o número da portaria referente à naturalização. Já em 547(86\%) fichas apresentavam o nome do município de nascimento; $276(34 \%)$ a unidade federativa do município de origem. Em 137(21,6\%) o número do cadastro do cartão do SUS estava preenchido.

Dos usuários cadastrados 420(66,0\%) eram do sexo feminino e $217(34,0 \%)$ do sexo masculino. A maior prevalência de idade foi entre 60 a 69 anos, com 180(28,2\%) usuários. Em seguida, encontram-se as faixas etárias de 50 a 59 e 70 a 79 anos de idade, 139(21,8\%) e 138(21,6\%), respectivamente. A média de idade foi de 63,9 anos.

No que se refere à escolaridade 295(46,3\%) dos usuários tinham ensino fundamental incompleto, seguido de 112(17,5\%) alfabetizados e 100(15,6\%) que não sabiam ler e escrever. No que se refere à raça 476(76,2\%) eram brancos, 39(6,1\%) pardos e $15(2,3 \%)$ negros. Quanto à situação familiar/ conjugal 188(29,5\%) informavam conviver com companheiro, com laços conjugais e sem filhos, $131(20,5 \%)$ conviviam com companheiro ou filhos; $73(11,4 \%)$ com familiares e sem filhos e $39(6,1 \%)$ sozinhos.

Documentos gerais, como o Cadastro de Pessoa Física (CPF), teve 546(85,7\%) dos campos preenchidos. Já o título, zona e série do título de 
eleitor tiveram dados preenchidos em 361(56,6\%), $352(55,2 \%)$ e $268(42 \%)$, respectivamente. Os outros documentos tiveram poucos campos preenchidos, como pode ser observado na Tabela 1.

Campos como nome do logradouro e código de endereçamento postal tiveram identificação expressiva de campos preenchidos com 594(93,2\%) e 500(78,4\%). Todavia, campos como bairro e tipo de logradouro tiveram frequência discreta, com 282(44,2\%) e 271(42,5\%),

Tabela 1 - Documentos gerais preenchidos na ficha do Programa HIPERDIA. Erval Seco-RS-Brasil, 2011

\begin{tabular}{|c|c|c|}
\hline Variáveis & $\mathbf{n}$ & $\%$ \\
\hline \multicolumn{3}{|c|}{ Número da CTPS* } \\
\hline Identificado & 06 & 0,9 \\
\hline \multicolumn{3}{|l|}{ Série da CTPS } \\
\hline Identificado & 07 & 1,0 \\
\hline \multicolumn{3}{|c|}{ Unidade Federativa da CTPS } \\
\hline Identificado & 8 & 1,2 \\
\hline \multicolumn{3}{|c|}{ Data da Emissão da CTPS } \\
\hline Identificado & 22 & 3,4 \\
\hline \multicolumn{3}{|c|}{ Número do PIS/PASEP** } \\
\hline Identificado & 04 & 0,6 \\
\hline
\end{tabular}

respectivamente. Em 67(10,5\%) e 144(22,6\%) havia a ocorrência do número do discagem direta à distância e telefone do usuário.

No campo dados clínicos do paciente verificou-se o registro dos fatores de risco e doenças, conforme a Tabela 2.

Em relação à presença de complicações pode-se verificar a ocorrência de doenças de predomínio na epidemiologia brasileira, como descrito na Tabela 3.

A partir desses dados é possível estratificar os riscos e prognósticos de pressão arterial sistêmica do usuário e suas características de saúde, conforme graus de risco e a probabilidade de algum evento cardiovascular nos próximos anos, aumentando exponencialmente, conforme os graus e, assim, estabelecer as condutas preconizadas pelo programa. A partir da altura e peso, pode-se, também, avaliar o Índice de Massa Corpórea (IMC). Assim, 304(47,7\%) dos usuários pertenciam ao grupo de peso normal e baixo risco para o desenvolvimento de comorbidades e
Tabela 2 - Fatores de risco e doenças concomitantes registrados na ficha do Programa HIPERDIA. Erval Seco-RS-Brasil, 2011

\begin{tabular}{|c|c|c|}
\hline Variáveis & $\mathbf{n}$ & $\%$ \\
\hline \multicolumn{3}{|c|}{ Diabetes Mellitus Tipo I } \\
\hline Sim & 105 & 16,4 \\
\hline Não & 479 & 75,2 \\
\hline Não Identificado & 53 & 8,4 \\
\hline \multicolumn{3}{|c|}{ Diabetes Mellitus Tipo II } \\
\hline Sim & 94 & 14,7 \\
\hline Não & 490 & 76,9 \\
\hline Não Identificado & 53 & 8,4 \\
\hline \multicolumn{3}{|l|}{ Tabagismo } \\
\hline Sim & 195 & 30,6 \\
\hline Não & 389 & 61,0 \\
\hline Não Identificado & 53 & 8,4 \\
\hline \multicolumn{3}{|l|}{ Sedentarismo } \\
\hline Sim & 256 & 40,1 \\
\hline Não & 328 & 51,5 \\
\hline Não Identificado & 53 & 8,4 \\
\hline \multicolumn{3}{|c|}{ Sobrepeso/Obesidade } \\
\hline Sim & 272 & 42,7 \\
\hline Não & 312 & 48,9 \\
\hline Não Identificado & 53 & 8,4 \\
\hline \multicolumn{3}{|c|}{ Hipertensão Arterial Sistêmica } \\
\hline Sim & 539 & 84,6 \\
\hline Não & 45 & 7,0 \\
\hline Não Identificado & 53 & 8,4 \\
\hline
\end{tabular}

$97(15,2 \%)$ pertenciam ao grupo de sobrepeso e de risco pouco aumentado. No grupo de obesidade $122(19,1 \%)$ foram classificados como obesos classe I, com risco moderado ao desenvolvimento de comorbidades, 52(8,1\%) como obesos classe II, e $10(1,5 \%)$ como obesos classe III, com risco grave e muito grave para desenvolver de comorbidades. Pode-se observar que 154(24,1\%) dos usuários apresentavam a medida de cintura entre $90 \mathrm{~cm}$ e 99 $\mathrm{cm}$, seguindo-se 135(21,1\%) com medidas de 100 cm e 109 cm e 96(15,7\%) com $110 \mathrm{~cm}$ e $119 \mathrm{~cm}$.

Todos os usuários cadastrados faziam uso de algum medicamento da tabela básica do programa HIPERDIA. Entre esses medicamentos informados estavam: Hidroclorotiazida (25mg), Propranolol (40mg), Captopril (25mg), Glibenclamida (5mg) e a Metformina (850mg). A glicemia foi informada 
Tabela 3 - Registro de complicações na ficha do Programa HIPERDIA. Erval Seco-RS-Brasil, 2011

\begin{tabular}{|c|c|c|}
\hline Variáveis & $\mathbf{n}$ & $\%$ \\
\hline \multicolumn{3}{|c|}{ Infarto Agudo do Miocárdio } \\
\hline Sim & 105 & 16,4 \\
\hline Não & 479 & 75,2 \\
\hline Não Identificado & 53 & 8,4 \\
\hline \multicolumn{3}{|c|}{ Outras Coronariopatias } \\
\hline Sim & 94 & 14,7 \\
\hline Não & 490 & 76,9 \\
\hline Não Identificado & 53 & 8,4 \\
\hline \multicolumn{3}{|c|}{ Acidente Vascular Cefálico } \\
\hline Sim & 195 & 30,6 \\
\hline Não & 389 & 61,0 \\
\hline Não Identificado & 53 & 8,4 \\
\hline \multicolumn{3}{|l|}{ Pé diabético } \\
\hline Sim & 256 & 40,1 \\
\hline Não & 328 & 51,5 \\
\hline Não Identificado & 53 & 8,4 \\
\hline \multicolumn{3}{|c|}{ Amputação por Diabetes Mellitus } \\
\hline $\operatorname{Sim}$ & 272 & 42,7 \\
\hline Não & 312 & 48,9 \\
\hline Não Identificado & 53 & 8,4 \\
\hline \multicolumn{3}{|l|}{ Doença Renal } \\
\hline Sim & 539 & 84,6 \\
\hline Não & 45 & 7,0 \\
\hline Não Identificado & 53 & 8,4 \\
\hline
\end{tabular}

em apenas 60(9\%) dos cadastros realizados, sendo 48(7,2\%) glicemia capilar de jejum, 12(1,8\%) pósprandial. Apenas $11(2 \%)$ dos usuários informaram fazer uso de insulina regularmente.

Em 543(85,2\%) formulários a data da consulta foi preenchida. O campo referente à assinatura do responsável pelo atendimento foi informado por apenas 392(61,5\%) das fichas. Estes campos são obrigatórios e identificam os responsáveis pelo cadastramento.

Em síntese os registros evidenciaram maior predominância de mulheres $(66,0 \%)$; na faixa etária de 60 a 69 anos (28,2\%); de cor branca $(76,2 \%)$; com ensino fundamental incompleto (46,3\%). Com relação aos dados clínicos verificouse a prevalência de pacientes com Diabetes Mellitus Tipo I (16,4\%); Diabetes Mellitus Tipo II $(14,7 \%)$ e Hipertensão Arterial (84,6\%). Quanto à ocorrência dos fatores de risco como o tabagismo com prevalência de $30,6 \%$, sedentarismo $(40,1 \%)$ e sobrepeso/obesidade $(42,7 \%)$, a presença de complicações como infarto agudo do miocárdio, acidente vascular cerebral doença renal e o uso de medicamentos disponíveis pelo programa.

\section{DISCUSSÃO}

A prevenção e cuidados à HAS e ao DM devem ocorrer prioritariamente na Atenção Primária ${ }^{(6)}$, porém quando dados gerais do usuário não estão disponíveis nas fichas de cadastro, conclui-se que há problemas na porta de entrada do sistema, o qual deveria desenvolver seu fazer de forma a organizar todas as ações em saúde e ter fluxo facilitado entre os níveis assistenciais.

Neste estudo os usuários cadastrados eram em maioria do sexo feminino. Em uma pesquisa desenvolvida em Pelotas-RS foi encontrado semelhante predomínio, com 69,9\% dos usuários do sexo feminino com HAS e um discreto predomínio masculino de $53,3 \%$ para o $\mathrm{DM}^{(4)}$. Isso pode ser atribuído à histórica existência de ações voltadas especificamente à saúde da mulher. Sendo assim, os profissionais devem incentivar indivíduos do sexo masculino a procurar os serviços de saúde, especialmente no que se refere às doenças crônico-degenerativas ${ }^{(7)}$.

Em relação à idade dos usuários, dados semelhantes foram encontrados em outro estudo, no qual a média foi de 63,5 anos $^{(8)}$. Isso traduz o predomínio dessas doenças em pessoas idosas(4,8-9), confirmando a necessidade de intensificar ações de promoção à saúde nas faixas etárias jovens, com hábitos de vida mais saudáveis e o controle das condições de saúde, repercutindo positivamente na senilidade. Além disso, a prevalência da HAS e DM aumenta com a idade dos indivíduos, soma-se a isto o considerável crescimento da população idosa no Brasil $^{(9)}$. Desse modo, gestores e profissionais de saúde devem se preocupar de imediato com essa parcela da população, desenvolvendo ações de controle e educação para a corresponsabilização pela sua saúde.

O predomínio de baixa alfabetização entre os usuários cadastrados é fator preocupante, pois são usuários com doenças crônicas que necessitam, muitas vezes, manter um nível de cuidado específico em relação a medicamentos 
e restrições alimentares ${ }^{(4)}$. Desse modo, eleva-se a responsabilidade das equipes de saúde em desenvolverem ações ativas, como grupos de saúde e visitas domiciliares, por exemplo, bem como facilitar o acesso das comunidades às ações de saúde e aos medicamentos necessários.

O predomínio da raça branca na população estudada pode ser explicado com base na colonização desta região, que é de origem germânica, polonesa e italiana. Apesar de não se conhecer, com exatidão, o impacto da miscigenação sobre a HAS no Brasil, esta é duas vezes mais prevalente em indivíduos de cor não branca ${ }^{(10)}$.

Expressivo número de fichas informava que os usuários conviviam com companheiro, com laços conjugais e sem filhos. Todavia, em estudo realizado em Maringá-Paraná a maioria dos pacientes residia com cônjuge e/ou filhos ${ }^{(7)}$. Ao analisar a faixa etária predominante com a situação familiar conjugal, sugere-se a hipótese de que os usuários são pessoas idosas que necessitam de estratégias de monitoramento domiciliar, sobretudo, para os cuidados de ESF.

Observou-se grande variação de frequências no registro de documentos gerais e obrigatórios. Isso gera um aspecto bastante peculiar, pois a informação dos documentos gerais não é obrigatória para o processamento final dos cadastros, o que ocorre de modo inverso aos documentos obrigatórios, como por exemplo, o número da identidade ${ }^{(11)}$. Quanto ao endereço do usuário, observou-se bom percentual de preenchimento, situação semelhante ao estudo realizado em Pelotas - RS, com elevado preenchimento do nome $(99,5 \%)$ e número $(98,4 \%)$ do logradouro e bairro $(97,9 \%)^{(12)}$.

Um campo fundamental da ficha de cadastramento do programa HIPERDIA pertence aos dados clínicos do paciente, com informações específicas para as ações dos profissionais de saúde, pois é possível categorizá-los e conhecer suas necessidades clínicas. Essa forma de classificação do usuário, conforme o risco, facilita a tomada de decisão tanto de controle, quanto de práticas de prevenção de agravos, especialmente porque se pode visualizar a pré-disposição de riscos à saúde. Por outro lado, essa classificação demanda profissionais habilitados, ou seja, que conheçam o formulário, os objetivos do programa e a finalidade de cada campo a ser preenchido.
Houve lacunas no preenchimento de informações de pressões arteriais diastólicas e sistólicas. Esse fato é preocupante uma vez que a sua mensuração é o primeiro passo para cadastrar o usuário no programa. Uma hipótese para o ocorrido é dos usuários apenas referiram ser hipertensos ou diabéticos sem a conferência do dado, o que não é adequado.

Os registros de informações sobre fatores de risco e doenças concomitantes e a presença de complicações colabora, significativamente, para qualificar a análise dos profissionais quanto à situação de saúde dos usuários. Esses dados são importantes, apesar de não obrigatórios, pois facilitam o enquadramento nos padrões de consenso nacional e internacional, além do acompanhamento futuro do usuário ${ }^{(11)}$. Por meio desses dados, os profissionais podem realizar a estratificação dos usuários, estabelecendo o tratamento considerado mais adequado a cada caso, pois o HIPERDIA orienta as ações dos profissionais a partir da classificação dos usuários em graus e ao risco de desenvolver algum evento cardiovascular nos próximos anos.

Nessa acepção, os achados revelam significativa frequência de usuários classificados em sobrepeso e obesidade. Assim, os resultados confirmaram clara magnitude do efeito do IMC sobre DM, HAS e dislipidemia ${ }^{(13)}$. Já, a medida da circunferência abdominal, mesmo não sendo campo obrigatório, favorece a classificação do indivíduo segundo as características prévias de risco ao desenvolvimento de doenças associadas $^{(11)}$.

Todos os usuários cadastrados faziam uso de algum medicamento da tabela básica do programa HIPERDIA. É importante destacar que, em muitos casos, o uso desses estava associado ao uso de outros medicamentos. No entanto, o uso da insulina como medicamento regular foi relatada em pequeno número. Todavia, não basta a existência dos medicamentos nos serviços de saúde, sendo necessário um uso consciente para que as ações não caminhem rumo ao uso indiscriminado de substâncias hipotensoras(14). É recomendado que os profissionais de saúde desenvolvam ações educativas, com incentivos às mudanças de hábitos e co-monitoramento da saúde com usuários participativos da sua condição clínica. 


\section{CONSIDERAÇÕES FINAIS}

No que diz respeito à efetividade dos registros nos cadastros identificou-se a inexpressividade no preenchimento de alguns campos, com isso observa-se que o programa precisaria passar por readequação no modelo das fichas de cadastros dos usuários. Campos pertencentes às ações de identificação do usuário, documentos gerais e obrigatórios precisam ser reavaliados segundo a sua relevância para efetivação das ações do programa.

Os resultados desse estudo ressalta a importância do correto preenchimento das fichas do programa, que precisam ser claras e precisas. Ainda, a importância de manter os sistemas de informação constantemente atualizados, gerando dados suficientes para que os profissionais de saúde e gestores possam conduzir as decisões de forma mais correta e coerente com a dinâmica de saúde local.

\section{REFERÊNCIAS}

1. Schimidt MI, Duncan BB, Silva GA, Menezes AM, Monteiro CA, Barreto SM, et al. Doenças Crônicas Não Transmissíveis no Brasil: Carga e desafios atuais. Séries. Online The Lancet Saúde no Brasil. [Internet] 2011 [acesso em 14 nov 2011]. Disponível: http://download.thelancet. com/flatcontentassets/pdfs/brazil/brazilpor4.pdf.

2. Organização Pan-Americana da Saúde (OPAS). Causas Principales de Mortalidad em las Américas. [acesso em 11 out 2011]. Disponível: http://ais.paho.org/phip/ viz/mort_causasprincipales_It_oms.asp

3. Ministério da Saúde (BR). Plano de Reorganização da atenção à Hipertensão Arterial e ao Diabetes Mellitus: Manual de Hipertensão Arterial e Diabetes Mellitus. Brasília; 2001.

4. Instituto Brasileiro de Geografia e Estatística - IBGE cidades [internet]. Dados básicos; [acesso em 4 out 2011]. Disponível: http://www.ibge.gov.br/cidadesat/ painel/painel.php?codmun=430730\#topo

5. Lima LM, Schwartz E, Mubiz RM, Zillmer JGV, Ludtke I. Perfil dos Usuários do HIPERDIA de Três Unidades Básicas de Saúde do Sul do Brasil. Rev. Gaúcha Enferm. 2011;32(2):323-9.

6. Organização Pan-Americana da Saúde (OPAS). Linhas de Cuidado: Hipertensão Arterial e Diabetes. Brasília. Organização Pan-Americana da Saúde; 2010.

7. Zavatini MA, Obreli-Neto PR, Cuman RKN. Estratégia Saúde da Família no tratamento de doenças crônico- degenerativas: avanços e desafios. Rev. Gaúcha Enferm. 2010; 31(4):647-54.

8. Cotta RMM, Batista KCS, Reis RS, Souza GA, Dias G, Castro FAF, et al. Perfil sociossanitário e estilo de vida de hipertensos e/ou diabéticos, usuários do Programa de Saúde da Família no município de Teixeiras, MG. Ciênc. saúde colet. 2009;14(4):1251-60.

9. Henrique NN, Costa OS, Vileti JL, Corrêa MCM, Carvalho EC. Hipertensão Arterial e Diabetes Mellitus: um estudo sobre os Programas de Atenção Básica. Rio de Janeiro. Rev. enferm. UERJ. 2008;16(2):168-73.

10. Sociedade Brasileira de Cardiologia/ Sociedade Brasileira de Hipertensão/ Sociedade Brasileira de Nefrologia. VI Diretrizes Brasileiras de Hipertensão Arterial. Online Arq. Bras. Cardiol. [Internet] 2010;95(1 supl.1):1-51 [acesso em 23 de nov de 2011]. Disponível: http://publicacoes.cardiol.br/consenso/2010/Diretriz_ hipertensao_associados.pdf.

11. Oliveira CA, Palha PF. Sistema de Informações Hiperdia, 2002-2004, adequação das informações. Cogitare enferm. 2008;13(3):395-402.

12. Zillmer JGV, Schwartz E, Muniz RM, Lima LM. Avaliação da completude das informações do Hiperdia em uma unidade básica do sul do Brasil. Rev. Gaúcha Enferm. 2010;31(2):240-6.

13. Gigante DP, Moura EC, Sardinha LMV. Prevalência de Excesso de Peso e Obesidade e Fatores Associados, Brasil, 2006. Rev. Saúde Públ. 2009;43(Supl 2):83-9.

14. Almeida GBS, Paz EPA, Silva GA. Representações sociais sobre hipertensão arterial e o cuidado: o discurso do sujeito coletivo. Acta Paul. Enferm. 2011;24(4):459-65. 